Cite as: Stephane H Maes, (2020), "Particles, Especially Virtual Particles, in a Multi-fold Universe vs. QFT", viXra:2010.0133v1, https://vixra.org/pdf/2010.0133v1.pdf, https://shmaesphysics.wordpress.com/2020/07/11/ particles-especially-virtual-particles-in-a-multi-fold-universe-vs-qft/, July 10, 2020.

\title{
Particles, Especially Virtual Particles, in a Multi-fold Universe vs. QFT
}

\author{
Stephane H. Maes ${ }^{1}$
}

July 10,2020

\begin{abstract}
:
In a multi-fold universe, gravity emerges from Entanglement through the multi-fold mechanisms. As a result, gravity-like effects appear in between entangled particles that they be real or virtual. Long range, massless gravity results from entanglement of massless virtual particles. Entanglement of massive virtual particles leads to massive gravity contributions at very smalls scales. Multi-folds mechanisms also result into a spacetime that is discrete, with a random walk fractal structure and non-commutative geometry that is Lorentz invariant and where spacetime nodes and particles can be modeled with microscopic black holes. All these recover General relativity at large scales and semi-classical model remain valid till smaller scale than usually expected. Gravity can therefore be added to the Standard Model. This can contribute to resolving several open issues with the Standard Model.

In this paper, we discuss the point of view of the virtual particles used to explain gravity emergence from entanglement and in particular position their use versus the more conventional view on virtual particles in QFT. Indeed, besides the fact that QFT has challenges to model particles, there are some strong views on what is or is not appropriate when it comes to involving virtual particles in conventional QFT, or vacuum fluctuations for that matter. The proposed multi-fold mechanisms on the other hand rely first and foremost on the concept of particles, with modifications to conventional QFT. In that context virtual particle play a central role. Besides evangelizing the need to evolve QFT, we also review how virtual particles are key to the notion of small scale non negligible addition of gravity to the standard model, and to a proposal for Ultimate Unification where al particles convey gravity and their proper interactions. We also discuss how this model is key and aligned to the area laws of blacks holes, Hawking's radiation and the absence of gravity shielding even when using virtual particle. This discussion will also offer some perspectives on QFT in curved spacetime. The bottom line is that there are no contradiction with the main views on virtual particles of conventional QFT proposed with multi-fold universe mechanisms and that in fact, while hard to formulate, the use of virtual particles could also be modeled with fields and associated multi-fold fields.
\end{abstract}

We also discuss comparing our model using pairs of entangled virtual particles versus models using only (or in addition) pairs of entangled gravitons. Such a multi-fold model with only gravitons may recover the same results or differ depending on how massive gravitons would be modeled in these new models. But we end up still recommending only a model where gravitons live in AdS(5).

\section{Introduction}

The new preprint [1] proposes contributions to several open problems in physics like the reconciliation of General Relativity (GR) with Quantum Physics, explaining the origin of gravity proposed as emerging from quantum (EPREinstein Podolsky Rosen) entanglement between particles, detailing contributions to dark matter and dark energy and explaining other Standard Model mysteries without requiring New Physics beyond the Standard Model other than the addition of gravity to the Standard Model Lagrangian. All this is achieved in a multi-fold universe that may well model our real universe, which remains to be validated.

\footnotetext{
${ }^{1}$ shmaes.physics@gmail.com
} 
With the proposed model of [1], spacetime and Physics are modeled from Planck scales to quantum and macroscopic scales and semi classical approaches appear valid till very small scales. In [1], it is argued that spacetime is discrete, with a random walk-based fractal structure, fractional and noncommutative at, and above Planck scales (with a 2-D behavior and Lorentz invariance preserved by random walks till the early moments of the universe). Spacetime results from past random walks of particles. Spacetime locations and particles can be modeled as microscopic blackholes (Schwarzschild for photons and spacetime coordinates, and metrics between Reisner Nordstrom [2] and Kerr Newman [3] for massive and possibly charged particles - the latter being possibly extremal). Although surprising, [1] recovers results consistent with other like [4], while also being able to justify the initial assumptions of black holes from the gravity or entanglement model in a multi-fold universe. The resulting gravity model recovers General Relativity at larger scale, as a 4-D process, with massless gravity, but also with massive gravity components at very small scale that make gravity significant at these scales. Semi-classical models also turn out to work well till way smaller scales that usually expected.

In this paper, we remain at a high level of discussion of the analysis and references are generic for the subjects. It makes the points accessible to a wider audience and keeps the door open to further papers or discussions devoted to details of interest. Yet, it requires the reader to review [1], as we do not revisit here all the details of the multifold mechanisms or reconstruction of spacetime. More targeted references for all the material discussed here are compiled in [1].

\section{Particles and QFT}

Quantum Field Theory (QFT) has a particle problem: it is often argued that the concept of particles does not exist or does not make sense in QFT on the basis that (See [1] for a more extensive discussion of some of these aspects):

- Particles can be created and annihilated all the time. So their number is always changing. It is by design of the second quantization and to handle particle to particle interactions. But as a result, typically, one does not identify or track a specific particle and definitively have problem with modeling particle to particle entanglement: QFT is more a statistical handling of particle behaviors and properties (hence also the close link to Statistical Physics, with which it shares many QFT tools and models).

- Particles are modeled by fields that have nonzero values over large regions of spacetime making it hard to know when a particle can be located somewhere versus somewhere else vs multiple particles.

- If a field or energy bump appears somewhere, it is hard to associate it to a particle as, with relativistic behaviors, it very rapidly spreads (all) over spacetime.

- Nonzero correlations of every spacetime point with every other spacetime, complicate particle interpretations

- In a curved spacetime, the curvature itself can create particles. The number of particles seen as present in a given spacetime region is not something that can typically be agreed upon by different observers [9].

Yet, particles exist and they are in fact central to some computation model of QFT like the Feynman diagrams. They are also observed, including in particle colliders like the LHC, where one experiment with the outcome of collisions and interactions modeled by these Feynman diagrams.

The dominant school of thought is that, in such cases, particles make sense asymptotically far from the interaction point(s), and, of course, that virtual particles are just intermediary representations of computational steps in Feynman diagrams and associated path integrals. See for example [5] for an opiniated discussions. Of course these statements are correct from the perspective of conventional QFT.

\section{Particles and Entanglement in Multi-fold Universes}




\subsection{Particles}

In a multi-fold universe, the notion of particle is central to deriving its core principles, focused on resolving the EPR paradox that involves entanglement between explicit particles. To address the problems with making sense of particles, [1] relies on a few principles:

- A universe where no supra-luminous interaction, movement, communications or signals are allowed.

- $\quad$ Path integrals must filter out, i.e. forbid, paths where any portion is spacelike, for another portion of the path.

- This is different from many conventional point of views in Quantum Physics, QFT or even New Physics. See for example [6].

This way, the problem of spacelike correlation and the spread and leak of the wavefunction is addressed. To this, [1] adds the recipe of knowingly tracking particles only between their creation and annihilation.

As explained in [1], when particles are entangled, an attractive (towards their center of mass) effective potential appears in between them with a gravity like behavior, function of their motion [17].

\subsection{Virtual Particles, Vacuum Fluctuations and Emerging Gravity}

[1] relies on the concepts of virtual particles from Feynman diagram and vacuum fluctuations to propose that energy sources (e.g. particles) emit pairs of entangled virtual particles in opposite direction. In our view, as discussed in next two paragraphs, it is aligned with conventional QFT and virtual particles as a mathematical tool [5]. At this stage, the important observation is that, doing so, and allowing virtual particles to contribute only as additional sources of entanglement in spacetime, results into activations of many multi-folds and as a result gravity like attractive potential towards the source or energy/particle [17]. This model recovers GR at large scale. The model is also not encountering problems with virtual particle shields that could, at first thought appear as gravity shield in multi-fold universe. They are not gravity shields, as is discussed in [8].

With respect to [5], we do know that virtual particles are even more controversial in conventional QFT than particles, and that usage of them may lead to further confusion and controversies. As argued, the proposal that we have for virtual particles (emitted in pairs by particles or by energy exciting the vacuum fluctuation), does not in our view counter the opiniated views presented for conventional QFT in [5]. Indeed, virtual particles are proposed here, have no associated creation operator or annihilation operators. They do not become real unless if there is a conventional reason for it (e.g. as in Casimir pressure [7]). Annihilation here is the recombination (possibly in the multi-folds) of a pair. Interaction is absorption by another system of the virtual particle and its entangled virtual partner at the exit point; not the result of creation or annihilation operators that would make the particle real. All conservation and Physics laws are respected for the virtual particles; except (or including depending on the point of view) energy conservation. The latter is viewed as respected with positive and negative energies allowed for durations specified by the uncertainty principles, the former allowed as temporary uncertainties. Of course this model can be seen also a mathematical trick whereby, there are fields of virtual particles and anti-particles (randomly distributed in the absence of other energy) and now with distribution of emitted (entangled) pairs dictated by energy distributions of all the other fields. The distribution spread from each source as time passes, at $\$ c \$$ for massless particles and at lower rates for massive particles; that also limit their range (not just due to the smaller speed but also due to the uncertainty principle; as is also the amount emitted when massive).

Entanglement between the pairs will create a "field" of multi-fold effects. All this could be formulated as a field, and we may do so in future work. However, it is not required for the rest of the analysis presented in this paper or in [1]; as, at the risk of incurring the ire of analyses like [5] for muddling the story, using the particle "tool" leads to easier ways to understand what is happening; yes, only if one does not get blocked by arguments like [5] that we do not dispute and in our view do not go against, despite possibly the first impression. In the rest of the paper, we assume that this is well understood and do not revisit the validity of explanations and models built on virtual 
particles: we know there is a pending field version if one wanted to go there. We urge the reader to take that point of view, from now on.

The ability to let entangled virtual particle pairs recombine at the exit points also explains what happens when virtual particle reach the limit of the range allowed by the uncertainty principle (or what happen when interaction disappears etc.); the pairs annihilate even if far away in spacetime; because they were entangled.

\subsection{Real vacuum pairs?}

If enough energy is available at a given spacetime location, when the energy is sufficient, real entangled pairs of particles and anti-particles can materialize. Situations like [9] are other examples. These are also some of the focus of [5]. If they are entangled, gravity like effects appear between these particles [1] and they are subjects to the multi-fold mechanisms of [1].

In multi-fold universe, the situation does not change. In general, there are no difference in terms of these use cases with conventional QFT. There is no reliance on real particle pairs created from vacuum fluctuations in what [1] proposes. The upcoming sections in the present paper will introduce such effects but it is to address other use cases and observations, proper to new, and different, aspects that we want to address.

\section{Particles and QFT in Curved Spacetime}

A priori, conventional QFT has such a track record of success that we can not dismiss its model as suitable in most situations. It is not intention in any case. However, we want however to point out that QFT story may not be that clear in curved spacetime, which, after all, is our everyday real universe. Indeed, it is well known that curved spacetime can create new particles and their number is not something not usually agreed upon by different observers [9]. It means that, in general, interaction (or scattering matrix) models can only make some sense when considering asymptotically flat Minkowski spacetime and counting all what happens to the particles in between from -infinity to + infinity time and space! Anything else is subjective and ambiguous, and that any notion of particle count is meaningless.

On one hand, the previous paragraph certainly adds to the argument that particles do not make sense in QFT. On the other hand, it may add to the view that QFT is reaching its limits in curved spacetime and in the presence of gravity.

In a multi-fold universe, the model goes as follow:

- $\quad$ The background spacetime is flat and Minkowski, without particle creations.

- Multifold mechanisms add effective potential contributions at every spacetime point or equivalently effective curvature.

- These are precursors to recovering GR at large enough scales.

- The model matches Einstein view that curvature is really just a geometrical visualization and modeling tool. It is a field on a flat space. His collaborator, Rosen, then tried to enshrine this view into evolutions of GR (see for example [10]).

- Because background spacetime is flat, QFT is to be expanded in that spacetime and should not suffer of the particle ambiguity encountered so far in curved spacetime. QFT in curved spacetime is a mathematical model to be understood as such: it allows to do computations. Many of its results are correct approximations. Some of its problems are reflections of the limitations of the model.

- The effective potential raises the energy around a spacetime location:

- When happening where real particles are located, the multi-fold mechanisms associates more production of entangled pairs of virtual particles (due to higher potential energy). This is also 
how [1] derives the area law for black hole entropy or how we can understand that there exist no gravity shields even when systems apparently block the virtual particles [8]. We do not count or track these virtual particles, only their multi-fold effects dictated by the source (energy and movement).

- When this additional (potential) energy is high enough, real entangled pairs of particles and antiparticles can be created. They are observable like any other particle. The number may not be agreed by different observer but we can very well explain; because different observers will not agree:

- When enough energy is reached to render such creation of real particle at a given spacetime point

- When, and in what order, they are created.

○ This explanation is much more "physical" and it is well aligned with GR.

With this viewpoint, we manage to have a better grasp on particles and again show that it is possible to make sense of them in the context of QFT. In particular, we are not limited to asymptotic discussions to model their interactions.

\section{Hawking's Radiation}

Let us make a digression that was in fact the original motivation for this paper. It started from reading the following popular science article [11], which argues that Hawking would have not presented a consistent explanation of black hole evaporation and his Hawking's radiation between its popular book [13] and his scientific publication where he first communicated his discovery [12]. The point here is not to dissect [11], but to emphasize the confusions that exists regarding all these notions, something also behind [5].

The first derivation, in [12], is an analysis of asymptotic scattering between past null infinity and future null infinity. It does not, and cannot, segregate between what happens on the black hole horizon and what happens outside around it (between it and future null infinity). In fact, it can't, because in the proposed model, the black hole is also, at the same time, too busy emerging from the star collapse in progress. The analysis relies on creation and annihilation operators, and so, in accordance with [5], it tracks real particle creation; not any virtual particles, as argued by [11].

However, then in [14, page 202] for example, Hawking provides a derivation at the black hole horizon, which anticipates [13], where Hawking argues that (entangled - he does not discuss that aspect) virtual pairs are created and one falls into the black hole, enabling the other one to become real. He also explains that it is just an intuitive visualization and refers to the derivation à [12] for the full proof.

How does this relates to the derivation in [1]? [1] derives a proportionality of the entropy of the horizon to its area by counting how many sources of gravity come from the fluctuations of the horizon (and it is not an evaporation argument). This is not re-questioned in [1] or [14]. However, [1] did not extracts exactly the constant of proportionality. $[12,14]$ estimates evaporation of the infinite asymptotic system seen from infinity so that the constant can be derived. It seems to imply that the number of particles created in between the black hole horizon and future null infinity is proportional to the area of the black hole horizon.

Is it true? A quick way to check if the model with multi-fold mechanism works is to estimate energy within a sphere as a function of the radius of a black hole (See [15 equation (7)]) and integrate over the radius. In the case of a Schwarzschild black hole, the result is indeed essentially in $r^{2}$, (integrated between the horizon and infinity) and hence proportional to the area of the horizon. So the conclusion is that, in a multi-fold universe, there is a contribution from the horizon effects and a contribution from the region beyond the horizon (between horizon and infinity). Each are proportional to the area of the horizon. Because at such scales, we recover GR, it is safe to deduct that the same is happening with QFT in curved spacetime. 
With respect to the arguments in [11], both reasonings lead to area proportionalities for the particles emitted. Only the asymptotic model ([12]) gives the correct proportionality constant ([13] gives a smaller contribution); but the intuitive picture of is easier to convey.

We consider that the above validates the predictions of multi-fold mechanisms with virtual particles when horizons or obstacles are introduced (e.g. [8])), as well as in what would conventionally be a curved spacetime. This is important to demonstrate consistency of the model proposed in [1].

\section{Gravitons vs. Virtual particles}

Note added 10/17/20: One should also note that in multi-fold universes as envisaged in [1], gravitons live in AdS(5) tangent to multi-fold spacetime and have effects in spacetime through effective potentials or curvature that can be also understood as graviton quasi particles $[18,19]$.

For completeness, it is important to understand that in [1], gravitons are associated to the multi-fold universe instead of being one of the virtual particles, or in lieu of the virtual particles. On one hand it is a question of definition as [1] shows that quantas in $\operatorname{AdS}(5)$, where multi-folds live correspond to quantas of spacetime in the spacetime. It is really motivated by the observation that doing so really provides complete symmetry between the multi-fold mechanisms in EPR entanglement and the entangled virtual pairs. Rejecting this approach and only invoking massless and possibly massive gravitons (produced as entangled pairs), could also work but may run with some of the problems met so far in QFT quantization of GR as well as may lose some of the arguments why gravity may not be negligible any more in some Standard Model phenomena, at least if massive gravitons were not also considered. Adding entangled virtual graviton pairs to the other entangled virtual particle pairs is of course also an option and that does not really change anything to [1].

So in a multi-fold universe graviton live outside of spacetime and have an effect on spacetime via the effective potentials (or effective curvatures). Doing so solves many problems of quantum gravity [1] and position nicely the real universe and superstrings [16]. But rebuilding the model with gravitons instead of virtual particle is fully supported by the approach. It leads to a lot of similar results and some variations. Detailing the implications could be the object of a future publication.

Note on 10/17/20: if we follow the idea that superstrings are consistent mathematical models that teach us about multi-fold universes (as well as the real universe) as reasoned in [20], then the fact that superstrings can only exists in universes with negative cosmological constant, and hence curvature, implies that, with multi-fold universes, graviton should be confined to AdS(5) [1,21], and have effects in spacetime that are: effective potentials, effective curvature or quasi particles. So the approach of [1] is the recommended way forward on that basis. It also ensures we have models behaving well at high energy.

\section{Conclusions}

We have discussed the reliance on particles and in particular virtual particles and vacuum fluctuations in [1]. To that effect we have justified its usage, arguing for better handling of particles in QFT, defending that virtual particles and vacuum fluctuation as used in [1] are not contradicting the spirit of [5] and showing the consistency of the approach in curved spacetime, especially for Hawking's Black hole radiation. [8] separately discussed with a similar reasoning when obstacles to virtual particles are introduced: gravity shielding does not take place.

We discussed also how virtual particles could be formulated as a field model; something that we have not done so far because it seems way more complicated and not the most pressing item to address to understand all the implications of multi-fold mechanisms. 
As this papers focuses on particles and virtual particles, we acknowledge that [1] could have selected to rely on spacetime gravitons (massless and massive) instead of any virtual pairs. In general, attributing gravity to entangled gravitons instead of other entangled pairs of virtual particles can be a theory equivalent to [1] but where gravitons also exist in spacetime. Outcome can be rigorously equivalent to [1] or differ depending on how massive gravitons would be considered. But not counting entanglement between other virtual pairs besides graviton seems arbitrary. Adding pairs of virtual gravitons to the other airs of entangled virtual particles is also an option and it does not change anything to [1]. Yet, we recommend a model based on [1] with gravitons living only in AdS(5).

References: (most references come from popular science to make the discussion more approachable)

[1]: Stephane H. Maes, (2020) “Quantum Gravity Emergence from Entanglement in a Multi-Fold

Universe", viXra:2006.0088v1, (June 9, 2020).

[2]: https://en.wikipedia.org/wiki/Reissner\%E2\%80\%93Nordstr\%C3\%B6m metric

[3]: https://en.wikipedia.org/wiki/Kerr-Newman metric

[4]: Burinskii, Alexander, (2008), "The Dirac-Kerr-Newman electron", arXiv:0507109v4

[5]: Arnold Neumaier, (2016), "Learn the Physics of Virtual Particles",

https://www.physicsforums.com/insights/physics-virtual-particles/

[6]: Ian H. Redmount, Wai-Mo Suen (1992). "Path integration in relativistic quantum mechanics", arXiv:gr-

qc/9210019v1

[7]: https://en.wikipedia.org/wiki/Casimir effect

[8]: Stephane H Maes, (2020), "No Gravity Shield in Multi-folds Universes", viXra:2010.0032v1

, https://shmaesphysics.wordpress.com/2020/06/26/no-gravity-shields-in-multi-folds-universes/, June 26, 2020.

[9]: Carroll, S., "Spacetime and Geometry. An Introduction to General Relativity", (Pearson Education Limited,

2014)

[10]: Rosen, Nathan (1973), "A bi-metric Theory of Gravitation", Gen. Rel. Grav., 4 (6): 435-447.

[11]: https://www.forbes.com/sites/startswithabang/2020/07/09/yes-stephen-hawking-lied-to-us-all-about-how-

black-holes-decay/

[12]: Hawking, S. (1974), "Black hole explosions?", Nature 248, 30-31.

[13]: Hawking, S. (1988), "A Brief History of Time", Bantam, 10th Anniversary edition.

[14]: Hawking, S. (1975), "Particle Creation by Black Holes", Commun. math. Phys. 43, 199-220.

[15]: Yuan K. Ha, (2005), "The Gravitational Energy of a Black Hole", arXiv:gr-qc/0508041

[16]: Stephane H Maes, (2020), "Dualities or Analogies between Superstrings and Multi-fold

Universe", viXra:2006.0178v1, https://shmaesphysics.wordpress.com/2020/06/14/dualities-or-analogies-betweensuperstrings-and-multi-fold-universes/, June 14, 2020.

[17]: Stephane H Maes, (2020), "Gravity-like Attractions and Fluctuations between Entangled

Systems?", viXra:2010.0010v1, https://shmaesphysics.wordpress.com/2020/06/25/gravity-like-attractions-and-

fluctuations-between-entangled-systems/, June 24, 2020.

[18]: Stephane H Maes, (2020), "Multi-fold Gravitons In-N-Out Spacetime",

https://shmaesphysics.wordpress.com/2020/07/27/multi-fold-gravitons-in-n-out-spacetime/, July 27, 2020,

(posted September 6, 2020)

[19]: Stephane H Maes, (2020), “Tracking Down The Standard Model With Gravity In Multi-Fold

Universes", https://shmaesphysics.wordpress.com/2020/08/30/tracking-down-the-standard-model-with-gravityin-multi-fold-universes/, August 20, 2020.

[20]: Stephane H Maes, (2020), "The E/G conjecture: entanglement is gravity and gravity is entanglement", https://shmaesphysics.wordpress.com/2020/10/15/the-e-g-conjecture-entanglement-is-gravity-and-gravity-isentanglement/, October 15, 2020.

[21]: Stephane H Maes, (2020), "Superstrings Encounter of the Second, Third or Fourth Types?", https://shmaesphysics.wordpress.com/2020/07/19/superstrings-encounter-of-the-second-third-orfourth-types/, July 5, 2020. 